\title{
Correction to: Uncovering the biosynthetic pathway of polysaccharide-based microbial flocculant in Agrobacterium tumefaciens F2
}

\author{
Shanshan $\mathrm{Pi}^{1} \cdot$ Jiguo $\mathrm{Qiu}^{2} \cdot{\text { Ang } \mathrm{Li}^{1} \cdot \text { Liang Feng }^{1} \cdot \operatorname{Dan} \mathrm{Wu}^{1} \cdot \text { He-ping Zhao }}^{3} \cdot$ Fang $\mathrm{Ma}^{1}$ \\ Published online: 4 September 2020 \\ (C) Springer-Verlag GmbH Germany, part of Springer Nature 2020
}

\section{Correction to: Applied Microbiology and Biotechnology} https://doi.org/10.1007/s00253-020-10850-2

In the original publication of the article, it was published under the title 'Applied microbiology and biotechnology uncovering the biosynthetic pathway of polysaccharide-based microbial flocculant in Agrobacterium tumefaciens F2'. The journal's name was included by mistake, the correct title is 'Uncovering the biosynthetic pathway of polysaccharidebased microbial flocculant in Agrobacterium tumefaciens F2'.

The online version of the original article can be found at https://doi.org/ $10.1007 / \mathrm{s} 00253-020-10850-2$

Ang Li

ang.li.harbin@gmail.com; li.ang@hit.edu.cn

Fang Ma

mafang@hit.edu.cn

1 State Key Laboratory of Urban Water Resource and Environment, School of Environment, Harbin Institute of Technology,

Harbin 150090, People's Republic of China

2 Key Laboratory of Agricultural Environmental Microbiology, Ministry of Agriculture, College of Life Sciences, Nanjing Agricultural University, Nanjing 210095, People's Republic of China

3 College of Environmental and Resource Sciences, Zhejiang University, Hangzhou 310058, People's Republic of China 